\title{
Antioxidant and antimicrobial capacities of Ganoderma lucidum
}

\begin{abstract}
The present study aimed to determine the total antioxidant status (TAS), total oxidant status (TOS), oxidative stress index (OSI) and antimicrobial activities of Ganoderma lucidum mushroom collected in Oguzeli region (Gaziantep province, Turkey). Rel Assay Diagnostics kits were used to determine TAS, TOS and OSI levels. Antimicrobial activity was determined using 9 different bacteria and fungi (Staphylococcus aureus, S. aureus MRSA, Enterococcus faecalis, Escherichia coli, Pseudomonas aeruginosa, Acinetobacter baumannii, Candida albicans, Candida krusei and Candida glabrata) using modified agar dilution method. The study findings demonstrated that $G$. lucidum had high antioxidant potential. Antimicrobial activity of the mushroom was also found to be normal. Thus, the consumption of G. lucidum as a natural source of antioxidants and an antimicrobial resource could be suggested.
\end{abstract}

Volume 7 Issue I - 2019

Celal B

Gaziantep University, Vocational School of Oguzeli, Turkey

Correspondence: Celal Bal, Gaziantep University, Vocational School of Oguzeli, Turkey, Tel +90 530 87676 II, Emailbal@gantep.edu.tr

Received: December 12, 2018 | Published: January 23, 2019

Keywords: Ganoderma lucidum, medicinal mushroom, antioxidant, oxidant, antimicrobial

\section{Introduction}

Mushrooms, one of the functional nutrients consumed for centuries, were always among the natural material rich in fiber, proteins, vitamins and minerals. In addition to their property as functional nutrients, several mushroom species possess natural pharmacological potential. Only a few studies were conducted on important pharmaceutical mushrooms even today, despite the fact that these studies reported ant proliferative, antimicrobial, and antioxidant, antitumor, antiallergic, hypoglycemic, anti-inflammatory and immune-enhancing properties of the investigated mushroom specie. ${ }^{1-5}$ Thus, it is not surprising that the interest in investigating the medicinal properties of wild mushrooms has increased over time.

Ganoderma lucidum, a cosmopolitan mushroom species, is a polypore rack mushroom that changes its color during growth until maturity from orange-white to bright red. There are both historical and contemporary research that supported the use of G. lucidum in various conditions including chronic inflammation and cancer. Its potent anti-oxidant and liver protective properties help slow the aging process, thus it is known as the "mushroom of immortality". The anti-cancer properties act as a powerful supplement in several malignancies, especially breast cancer and lymphoma, and recent studies demonstrated surprising safety profile in these cases. ${ }^{6-9}$

The present study aimed to determine the total antioxidant status, total oxidant status and oxidative stress index of Ganoderma lucidum (Curtis) P. Karst mushroom collected in Gaziantep province Oguzeli region (Turkey) in order to identify the antioxidant capacity of the mushroom.

\section{Material and Method}

G. lucidum mushrooms were collected in Gaziantep province (Oguzeli region) in Turkey (Figure 1). The samples were transported to the laboratory environment under adequate conditions and extracted with methanol $(\mathrm{MeOH})$ and dichloromethane $(\mathrm{DCM})$ in Soxhlet extractor (Gerhardt EV14). The extracts were concentrated in a rotary evaporator (Heidolph Laborota 4000 Rotary Evaporator).

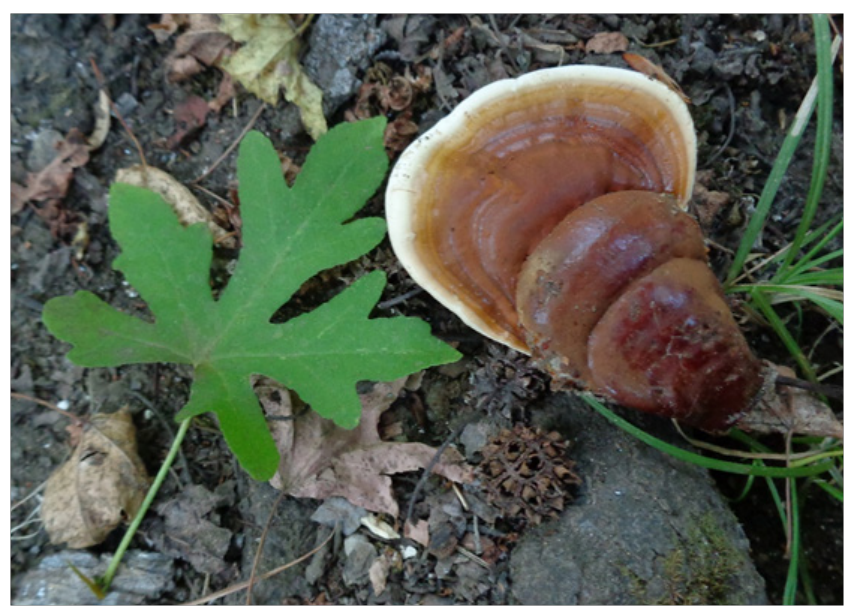

Figure I Ganoderma lucidum (Curtis) P. Karst.

The identified $30 \mathrm{~g}$ samples were extracted in Soxhlet extractor for about 6hours at $50^{\circ} \mathrm{C}$ with methanol $(\mathrm{MeOH})$ and dichloromethane (DCM) (Gerhardt EV14). The obtained extracts were concentrated with a rotary evaporator (Heidolph Laborota 4000Rotary Evaporator).

\section{TAS, TOS and OSI tests}

Rel Assay brand commercial kits were used to determine $G$. lucidum mushroom TAS, TOS and OSI levels. Trolox was used as the calibrator in the TAS tests and hydrogen peroxide was used as the calibrator in TOS tests. ${ }^{10,11}$ When calculating the OSI value, TAS and TOS units were equalized and the proportion of the two values was calculated. Thus, the OSI percentage value was calculated. ${ }^{11}$

\section{Antimicrobial Activity Tests}

Antimicrobial activity assays on mushroom $\mathrm{MeOH}$ and DCM extracts were conducted with the agar dilution method as recommended by the Clinical and Laboratory Standards Institute (CLSI) and the European Committee on Antimicrobial Susceptibility Testing (EUCAST). Minimal inhibitory concentrations (MIC) for 
each extract were tested against standard bacterial and fungal strains Staphylococcus aureus ATCC 29213, Staphylococcus aureus MRSA ATCC 43300, Enterococcus faecalis ATCC 29212, Escherichia coli ATCC 25922, Pseudomonas aeruginosa ATCC 27853, Klebsiella pneumoniae ATCC 700603, Acinetobacter baumannii ATCC 19606, Candida albicans ATCC 10231, Candida krusei ATCC 34135 ATCC 13803, and Candida glabrata ATCC 90030 were used as test microorganisms. Bacterial strains were pre-cultured in Muller Hinton Broth medium and fungal strains were pre-cultured in RPMI 1640Broth medium. The turbidity of bacteria and fungi was prepared according to McFarland 0.5 scale to obtain a standard inoculum. All extracts were tested at $800-12.5 \mu \mathrm{g} / \mathrm{ml}$ concentrations and all dilutions were conducted with distilled water. The solvents used in the extracts were tested lean for antimicrobial activity. Fluconazole and amphotericin were used as reference drugs for B fungi and Amikacin, Ampicillin and Ciprofloxacin were used as reference drugs for bacteria. The lowest dilution that inhibited the propagation of bacteria and fungi was determined as the minimal inhibitory concentration (MIC). ${ }^{12-17}$

\section{Results and discussion}

\section{TAS,TOS, and OSI}

In the present study, G. lucidum mushroom TAS, TOS and OSI values were determined for the first time in literature. The findings are presented in Table 1.

Table I G. lucidum TAS, TOS and OSI values

\begin{tabular}{llll}
\hline & TAS & TOS & OSI \\
\hline G. lucidum & $5.509 \pm 0.198$ & $10.177 \pm 0.116$ & $0.185 \pm 0.008$
\end{tabular}

No data are available in the literature for the TAS, TOS and OSI

Table 2 Antimicrobial Activity of G. lucidum

\begin{tabular}{|c|c|c|c|c|c|c|c|c|c|}
\hline & S. aureus & S. aureus MRSA & E. faecalis & E. coli & P. aeruginosa & A. baumannii & C. albicans & C. glabrata & C. krusei \\
\hline DCM & 200 & 200 & 100 & 200 & 200 & 100 & 100 & 100 & 100 \\
\hline $\mathrm{MeOH}$ & 200 & 200 & 100 & 100 & 200 & 100 & 50 & 100 & 50 \\
\hline Ampicillin & 1.56 & 3.12 & 1.56 & 3.12 & 3.12 & - & - & - & - \\
\hline Amikacin & - & - & - & 1.56 & 3.12 & 3.12 & - & - & - \\
\hline Ciprofloxacin & 1.56 & 3.12 & 1.56 & 1.56 & 3.12 & 3.12 & - & - & - \\
\hline Fluconazole & - & - & - & - & - & - & 3.12 & 3.12 & - \\
\hline Amphotericin B & - & - & - & - & - & - & 3.12 & 3.12 & 3.12 \\
\hline
\end{tabular}

The MIC values are presented in units of $\mu \mathrm{g} / \mathrm{mL}$.

It was determined that mushroom $\mathrm{MeOH}$ extracts were effective on test microorganisms at $50-200 \mu \mathrm{g} / \mathrm{mL}$ concentrations. Furthermore, it was found that mushroom DCM extracts were effective on test microorganisms at concentrations of $100-200 \mu \mathrm{g} / \mathrm{mL}$. Previous studies demonstrated that different concentrations of $G$. lucidum aqueous extracts were effective on Bacillus anthracis, B. cereus, B. subtilis, Micrococcus luteus, Staphylococcus aureus, Escherichia coli, Klebsiella oxytoca, K. pneumoniae, Proteus vulgaris, Salmonella tompson, S. typhi, S. typhimurium and Serratia marcescens. ${ }^{22}$ It was reported that different concentrations of G. lucidum $\mathrm{MeOH}$ extracts were effective on S. aureus, B. cereus Listeria monocytogenes, Micrococcus flavus, P. aeruginosa, E. coli, S. typhimurium and Enterobacter cloacae. In the present study, $\mathrm{MeOH}$ and DCM extracts of G. lucidum were used. It was determined that the extracts exhibited antimicrobial effects in different concentrations on $S$. aureus, $S$. values of G. lucidum mushroom. However, in studies conducted on the TAS, TOS and OSI values with different mushroom species, it was determined that the TAS values of Auricularia auricula, Trametes versicolor, Lepiota cristata, Leucoagaricus leucothites, Cyclocybe cylindracea and Paxillus involutus mushrooms were 1.010, 0.820, $2.210,8.291,4.325$ and $1.230 \mathrm{mmol} / \mathrm{L}$, respectively, and TOS values were $23.910,17.760,24.357,10.797,21.109$ and $7.533 \mu \mathrm{mol} / \mathrm{L}$ and OSI values were reported as $2.367,2.166,1.103,0.130,0.488$ and 0.613 , respectively. ${ }^{4,18-21}$ Compared to the above-mentioned studies, it was determined that the TAS value of G. lucidum was lower when compared to L. leucothites mushroom and higher when compared to that of A. auricula, T. versicolor, L. cristata, C. cylindracea and $P$. involutus mushrooms. It was determined that the TOS value of $G$. lucidum was a lower when compared to L. leucothites, A. auricula, T. versicolor, L. cristata and C. cylindracea mushrooms and higher when compared to P. involutus mushroom. It was determined that the OSI value of G. lucidum was higher when compared to L. leucothites mushroom and lower when compared to that of A. auricula, T. versicolor, L. cristata, C. cylindracea and $P$. involutus mushrooms.

It was suggested that the above-mentioned differences in TAS, TOS and OSI values were due to differences among mushroom species and environmental and inherent factors such as differences between the habitats and the substrates the mushrooms utilize. Thus, it was determined that G. lucidum has a high antioxidant potential and can be consumed as a natural antioxidant source.

\section{Antimicrobial Activity Tests}

In the present study, the lowest extract concentrations that prevented the proliferation of test microorganisms were determined and the findings are presented in Table 2. aureus MRSA, E. faecalis, E. coli, P. aeruginosa, A. baumannii, C. albicans, $C$. glabrata and $C$. krusei test microorganisms. Thus, it was concluded that $G$. lucidum was a natural antimicrobial agent against tested microorganisms.

\section{Conclusion}

In the present study, antioxidant and antimicrobial activities of wild G. lucidum mushroom were determined. In conclusion, it was determined that the mushroom exhibited high antioxidant activity. It could also be consumed as a good antimicrobial agent against tested microorganisms.

\section{Acknowledgments}

I would like to express our gratitude to Dr. Mustafa SEVINDIK and Dr. Hasan AKGUL for their contributions to the present study. 


\section{Conflicts of Interest}

No conflict of interest was declared by the authors.

\section{References}

1. Quereshi S, Pandey AK, \& Sandhu SS. Evaluation of antibacterial activity of different Ganoderma lucidum extracts. J Sci Res. 2010;3:9-13.

2. Rezaeian S, Saadatm S, Sattari TN, et al. Antioxidant potential and other medicinal properties of edible mushrooms naturally grown in Iran. Biomedical Research. 2016;27(1):240-247.

3. Y1lmaz A, Yildiz S, Kilıc C, et al. Total phenolics, flavonoids, tannin contents and antioxidant properties of Pleurotus ostreatus cultivated on different wastes and sawdust. International Journal of Secondary Metabolite. 2016;4(1):1-9.

4. Akgul H, M Sevindik, C Coban, et al. New Approaches in Traditional and Complementary Alternative Medicine Practices: Auricularia auric$u l a$ and Trametes versicolor. J Tradit Med Clin Natur. 2017;6(239):2.

5. Sevindik M. Investigation of Antioxidant/Oxidant Status and Antimicrobial Activities of Lentinus tigrinus. Advances in Pharmacological Sciences. 2018:1-4.

6. Guler G, Himmetoglu C, Jimenez RE, et al. Aberrant expression of DNA damage response proteins is associated with breast cancer subtype and clinical features. Breast cancer research and treatment, 2011;129(2):421-432.

7. Wachtel GS, Yuen J, Buswell JA, et al. Ganoderma lucidum (Lingzhi or Reishi). Herbal Medicine: Biomolecular and Clinical Aspects. 2nd ed. 2011.

8. Jin X, Ruiz BJ, Sze DM, et al. Ganoderma lucidum (Reishi mushroom) for cancer treatment. Cochrane Database Syst Rev. 2012;6:1-37.

9. Barbieri A, Quagliariello V, Del VV, et al. Anticancer and anti-inflammatory properties of Ganoderma lucidum extract effects on melanoma and triple-negative breast cancer treatment. Nutrients. 2017;9(3):210.

10. Erel O. A novel automated direct measurement method for total antioxidant capacity using a new generation, more stable ABTS radical cation. Clinical biochemistry. 2004;37(4):277-285.

11. Erel O. A new automated colorimetric method for measuring total oxidant status. Clinical biochemistry. 2005;38(12):1103-1111.
12. Bauer AW, Kirby WMM, Sherris JC, et al. Antibiotic susceptibility testing by a standardized single disk method. American journal of clinical pathology. 1966;45(4):493-496.

13. Hindler J, Hochstein L, Howell A. Preparation of routine media and reagents used in antimicrobial susceptibility testing. American Society for Microbiology. 1992:5-14.

14. David WH, Diane MC, Joanne Dx, et al. Methods for Antimicrobial Susceptibility Testing of Anaerobic Bacteria; Approved Standard 8th ed. CLSI. 2012;32(5):8-11.

15. Matuschek E, Brown DF, Kahlmeter G. Development of the EUCAST disk diffusion antimicrobial susceptibility testing method and its implementation in routine microbiology laboratories. Clinical Microbiology and Infection. 2014;20(4):255-266.

16. Christian Giske. Breakpoint Tables for Bacteria Interpretation of MICs and Zone Diameters, EUCAST. European Committee on Antimicrobial Susceptibility Testing. 2018-12-24.

17. Sevindik M. Heavy metals content and the role of Lepiota cristata as antioxidant in oxidative stress. J Bacteriol Mycol. Open Access 2018;6(4):237-239.

18. Sevindik M, Akgul H, Dogan M, et al. Determination of antioxidant, antimicrobial, DNA protective activity and heavy metals content of Laetiporus sulphureus. Fresenius Environmental Bulletin. 2018;27(3):1946-1952.

19. Sevindik M, Rasul A, Hussain G, et al. Determination of anti-oxidative, anti-microbial activity and heavy metal contents of Leucoagaricus leucothites. Pak J Pharm Sci. 2018;31(5):2163-2168.

20. Çolak OF, Rasul A, Sevindik M. A study on Paxillus involutus: total antioxidant and oxidant potential. Turkish Journal of Life Sciences. 2018;3(2):244-247.

21. Yoon SY, Eo SK, Kim YS, et al. Antimicrobial activity of Ganoderma lucidum extract alone and in combination with some antibiotics. Archives of pharmacal research, 1994;17(6):438-442.

22. Heleno SA, Ferreira IC, Esteves AP, et al. Antimicrobial and demelanizing activity of Ganoderma lucidum extract, p-hydroxybenzoic and cinnamic acids and their synthetic acetylated glucuronide methyl esters. Food and chemical toxicology. 2013;58: 95-100. 